\title{
Quarkonia results in heavy-ion collisions from CMS
}

\author{
Vineet Kumar* \\ (For CMS Collaboration) \\ Nuclear Physics Division \\ Bhabha Atomic Research Center \\ Mumbai, India \\ E-mail: Vineet.Kumarecern.ch
}

\begin{abstract}
This writeup summarizes CMS results on quarkonia measurements in $\mathrm{pp}, \mathrm{pPb}$ and $\mathrm{PbPb}$ collisions at LHC. CMS has excellent muon detection capabilities which has resulted in a wealth of results on quarkonia (both charmonia as well as bottomonia) measured in dimuon channel. The good mass resolution in dimuon channels allows precise measurement of all three $\Upsilon$ states and their relative yields in $\mathrm{pp}, \mathrm{pPb}$ as well as $\mathrm{PbPb}$ systems, which have ability to quantify the properties of strongly interacting matter. In the charmonia sector, measurements of relative yields of $\mathrm{J} / \psi$, $\psi(2 S)$ are equally useful. In addition excellent vertex capability of CMS enables measurement of B mesons via its decay to $\mathrm{J} / \psi$ which are useful tool to verify energy loss mechanisms of heavy quarks in medium. An overview of these measurements is given. How these measurements compare with other experiments at RHIC and LHC and have improved the understanding of heavy ion collisions has been discussed.
\end{abstract}

7th International Conference on Physics and Astrophysics of Quark Gluon Plasma

1-5 February, 2015

Kolkata, India

${ }^{*}$ Speaker. 


\section{Introduction}

The goal of the SPS, RHIC, and LHC heavy-ion programmes is to validate the existence and study the properties of the quark-gluon plasma (QGP), a state of deconfined quarks and gluons. One of its most striking expected signatures is the suppression of quarkonium states [1], both of the charmonium $\left(\mathrm{J} / \psi, \psi(2 \mathrm{~S}), \chi_{c}\right.$, etc.) and the bottomonium $\left(\Upsilon(1 \mathrm{~S}, 2 \mathrm{~S}, 3 \mathrm{~S}), \chi_{b}\right.$, etc.) families. This is thought to be a direct effect of deconfinement, when the binding potential between the constituents of a quarkonium state, a heavy quark and its antiquark, is screened by the colour charges of the surrounding light quarks and gluons. The suppression is predicted to occur above the critical temperature of the medium $\left(\mathrm{T}_{c}\right)$ and depends on the $\mathrm{Q} \overline{\mathrm{Q}}$ binding energy alternatively the suppression can be understood in terms of quarkonium dissociation by collisions with gluons [2,3].

The first such measurement was the 'anomalous' $\mathrm{J} / \psi$ suppression discovered in $\mathrm{PbPb}$ collisions at $\sqrt{s_{N N}}=17.3 \mathrm{GeV}$ at the SPS, which was considered as a hint of QGP formation. The RHIC measurements in AuAu at $\sqrt{s_{N N}}=200 \mathrm{GeV}$ [4] showed almost the same suppression at a much higher energy contrary to the expectation [5]. Such an observation was consistent with the scenario that at higher collision energy the expected greater suppression is compensated by regeneration of $\mathrm{J} / \psi$ by recombination of two independently produced charm quarks [6]. Since the LHC first performed $\mathrm{Pb}+\mathrm{Pb}$ collisions at $\sqrt{s_{N N}}=2.76 \mathrm{TeV}$, a wealth of quarkonia results have become available [7, 8]. Some of these results are presented in this writeup and their significance is discussed. The quarkonia yields in heavy-ion collisions can also be modified by non-QGP effects such as, modification of the parton distribution functions inside the nucleus, known as shadowing and dissociation due to hadronic or comover interaction $[9,10]$. To get a quantitative idea about these effects, quarkonia measurements in $\mathrm{pPb}$ collisions at $\sqrt{s_{N N}}=5.02 \mathrm{TeV}$ are performed. These measurements are also discussed in this writeup.

\section{CMS detector at LHC}

The central feature of CMS is a superconducting solenoid, of $6 \mathrm{~m}$ internal diameter, providing a field of 3.8T Within the field volume are the silicon pixel and strip tracker, the crystal electromagnetic calorimeter (ECAL) and the brass/scintillator hadron calorimeter (HCAL). Muons are measured in gas-ionization detectors embedded in the steel return yoke. In addition to the barrel and endcap detectors, CMS has extensive forward calorimetry. The muons are measured in the pseudorapidity window $|\eta| \leq 2.4$, with detection planes made of three technologies: Drift Tubes, Cathode Strip Chambers, and Resistive Plate Chambers. Matching the muons to the tracks measured in the silicon tracker results in a transverse momentum resolution better than $1.5 \%$ for $\mathrm{p}_{T}$ smaller than $100 \mathrm{GeV} / \mathrm{c}$. A much more detailed description of CMS can be found in ref. [11]. Table 1 shows all the systems, center of mass energies and collected integrated luminosities for the measurements described in the writeup.

\section{Measurements of charmonia states at CMS}

The CMS experiment carries out $\mathrm{J} / \psi$ measurements at high transverse momentum $\left(p_{T}>6.5\right.$ $\mathrm{GeV} / c$ ) and in the rapidity range $|y| \leq 2.4$. Figure 1 (left) shows the nuclear modification factor $\left(R_{A A}\right)$ of $\mathrm{J} / \psi$ in $\mathrm{PbPb}$ collisions at $\sqrt{s_{N N}}=2.76 \mathrm{TeV}$ as a function of number of participants 
Table 1: LHC runs of heavy ion intrest.

\begin{tabular}{cccl} 
year & system & $\sqrt{s_{\mathrm{NN}}}(\mathrm{TeV})$ & $L_{\text {int }}$ \\
\hline 2010 & $\mathrm{~Pb}-\mathrm{Pb}$ & 2.76 & $\sim 10 \mu \mathrm{b}^{-1}$ \\
2011 & $\mathrm{pp}$ & 2.76 & $\sim 250 \mathrm{nb}^{-1}$ \\
2011 & $\mathrm{~Pb}-\mathrm{Pb}$ & 2.76 & $\sim 150 \mu \mathrm{b}^{-1}$ \\
2013 & $\mathrm{p}-\mathrm{Pb}$ & 5.02 & $\sim 30 \mathrm{nb}^{-1}$ \\
2013 & $\mathrm{pp}$ & 2.76 & $\sim 5 \mathrm{pb}^{-1}$
\end{tabular}

(centrality) measured by CMS $[12,13]$. The nuclear modification factor of these high $p_{T}$ prompt $\mathrm{J} / \psi$ decreases with increasing centrality showing moderate suppression even in the most peripheral collisions. By comparing with the STAR results [14] at RHIC it follows that the suppression of high $p_{T} \mathrm{~J} / \psi$ has increased with collision energy. The ALICE J/ $\psi$ results [15] cover low $p_{T}$ range
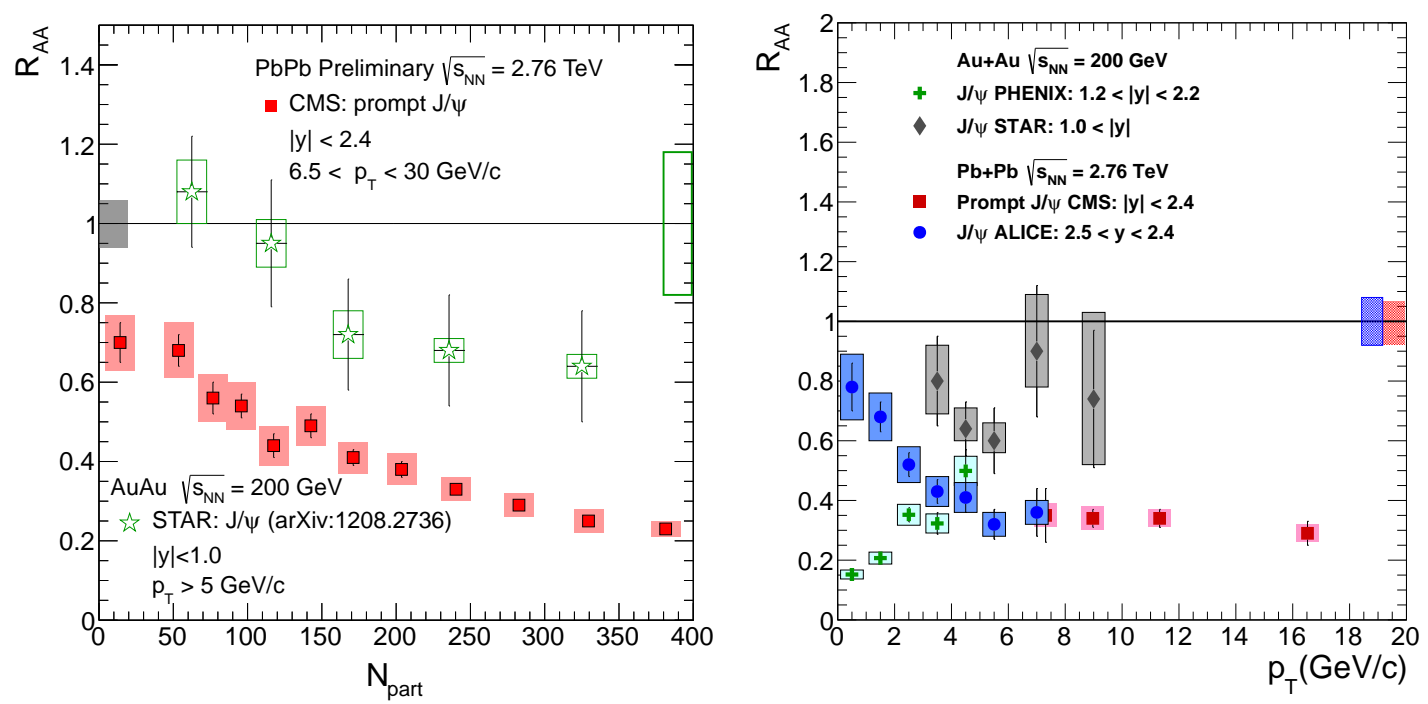

Figure 1: The nuclear modification factor $\left(R_{\mathrm{AA}}\right)$ of $\mathrm{J} / \psi$ in $\mathrm{PbPb}$ collisions at $\sqrt{s_{N N}}=2.76 \mathrm{TeV}$ as a function of number of participants (left) and $\mathrm{p}_{T}$ (right), measured by CMS experiment [12,13]. Measurements from other detectors are also shown for comparison.

at forward rapidity. At low $\mathrm{p}_{T}$ the $R_{A A}$ has little or no centrality dependence except very peripheral collisions.

Figure 1 (right) shows $R_{A A}$ of $\mathrm{J} / \psi$ in $\mathrm{PbPb}$ collisions at $\sqrt{s_{N N}}=2.76 \mathrm{TeV}$ as a function of $p_{T}$ measured by CMS, ALICE and PHENIX experiments. The $R_{A A}$ is found to be nearly independent of $p_{T}$ (above $6.5 \mathrm{GeV} / c$ ) showing that $\mathrm{J} / \psi$ remains suppressed even at very high $p_{T}$, up to $\sim 16$ $\mathrm{GeV} / c[12,13]$. By comparing with the STAR results [14] at RHIC it follows that the suppression of high $p_{T} \mathrm{~J} / \psi$ has increased with collision energy. The ALICE J/ $\psi$ data [15] shows that $R_{A A}$ increases with decreasing $p_{T}$ below $4 \mathrm{GeV} / c$. On comparing with the PHENIX forward rapidity measurement [4], it can be said that low $\mathrm{p}_{T} \mathrm{~J} / \psi$ at LHC are enhanced in comparison to RHIC. These observations suggest regeneration of $\mathrm{J} / \psi$ at low $p_{T}$ by recombination of independently produced 
charm pairs. Another hint of regeneration is given by CMS measurement of ratios of charmonia in $\mathrm{PbPb}$ and pp collisions. Figure 2 (left) shows the double ratio of $\psi(2 \mathrm{~S})$ and $\mathrm{J} / \psi$ as a function of centrality measured by CMS in two kinematic regions [16]. In the midrapidity bin, where all measured charmonia must have $\mathrm{p}_{T}>6.5 \mathrm{Gev} / c$, the double ratio is less than unity in all three centrality bins, with a central-integrated value of $0.45 \pm 0.13$ (stat.) \pm 0.07 (syst.), including the global pp uncertainties. In the forward rapidity bin, which includes charmonia with $\mathrm{p}_{T}$ extending down to $3 \mathrm{GeV} / c$, the double ratio increases with centrality, reaching the value $2.31 \pm 0.53$ (stat.) \pm 0.38 (syst.) \pm 0.15 (pp) in the most central collisions. It indicates that central $\mathrm{PbPb}$ collisions produce more $\psi(2 \mathrm{~S})$ mesons than $\mathrm{J} / \psi$ mesons, with respect to the pp reference.

Additional insight into properties of the QGP can be obtained through study of the azimuthal anisotropy of quarkonia in lead-lead ( $\mathrm{PbPb}$ ) collisions. A non-zero elliptic anisotropy, for example, could provide evidence for recombination of thermalized charm quarks. Figure 2 (right) shows CMS measurement of prompt $\mathrm{J} / \psi \mathrm{v}_{2}$ [17] as a function of $\mathrm{J} / \psi$ transverse momentum in $10-60 \%$ centrality events. The results are compatible with a $\mathrm{p}_{T}$ independent, non zero anisotropy, whether measured at low- $\mathrm{p}_{T}\left(3<\mathrm{p}_{T}<6.5 \mathrm{GeV} / c\right)$ in the forward rapidity interval $1.6<|y|<2.4$, or at high- $\mathrm{p}_{T}\left(6.5<\mathrm{p}_{T}<30 \mathrm{GeV} / c\right)$ in rapidity interval $|y|<2.4$.
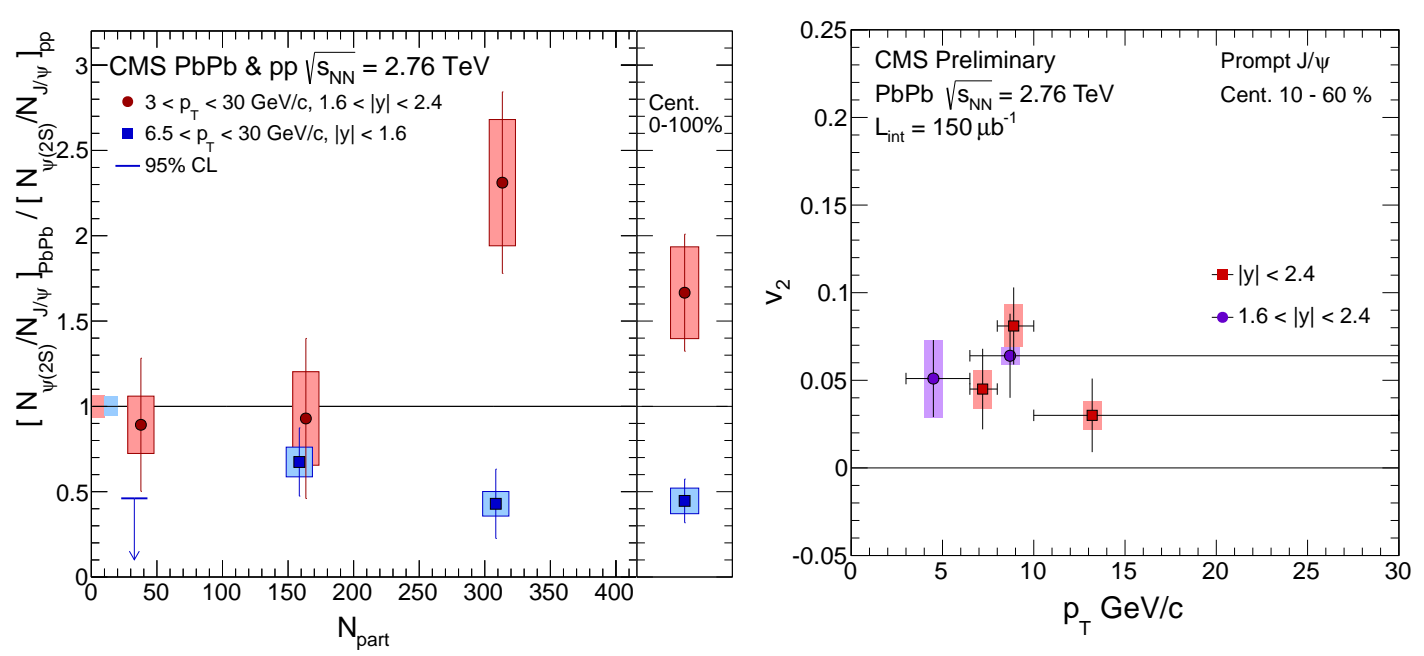

Figure 2: (left) Double ratio of prompt $\psi(2 \mathrm{~S})$ and $\mathrm{J} / \psi$ as a function of centrality measured by CMS in two kinematic regions [16]. (right) Prompt $J / \psi$ azimuthal anisotropy $\left(\mathrm{v}_{2}\right)$ as a function of $\mathrm{J} / \psi$ transverse momentum [17].

\section{Measurements of bottomonia states at CMS}

At LHC energies, the $\Upsilon$ states are produced with good statistics. The CMS measurements [18, 19] reveal that the higher $\Upsilon$ states, $\Upsilon(2 S)$ and $\Upsilon(3 S)$, are more suppressed relative to the ground state $\Upsilon(1 \mathrm{~S})$, a phenomenon known as sequential suppression. Figure 3(left) shows the $R_{A A}$ of $\Upsilon(1 \mathrm{~S})$ and $\Upsilon(2 \mathrm{~S})$ measured by CMS. The figure also shows STAR measurement of $\Upsilon(1 \mathrm{~S}) R_{A A}$ [20]. We can clearly see from the figure that $\Upsilon$ 's are more suppressed at higher collision energy. Figure 3(right) shows the $R_{A A}$ of $\Upsilon(1 \mathrm{~S})$ and $\Upsilon(2 S)$ measured by CMS along with the ALICE measurements [21] 
at forward rapidity, $\left(2.5 \leq y^{\Upsilon} \leq 4.0\right)$. The figure indicate that at forward rapidity, $\Upsilon(1 S)$ is slightly more suppressed than the CMS measurements at midrapidity, $\left|y^{\Upsilon}\right| \leq 2.4$.
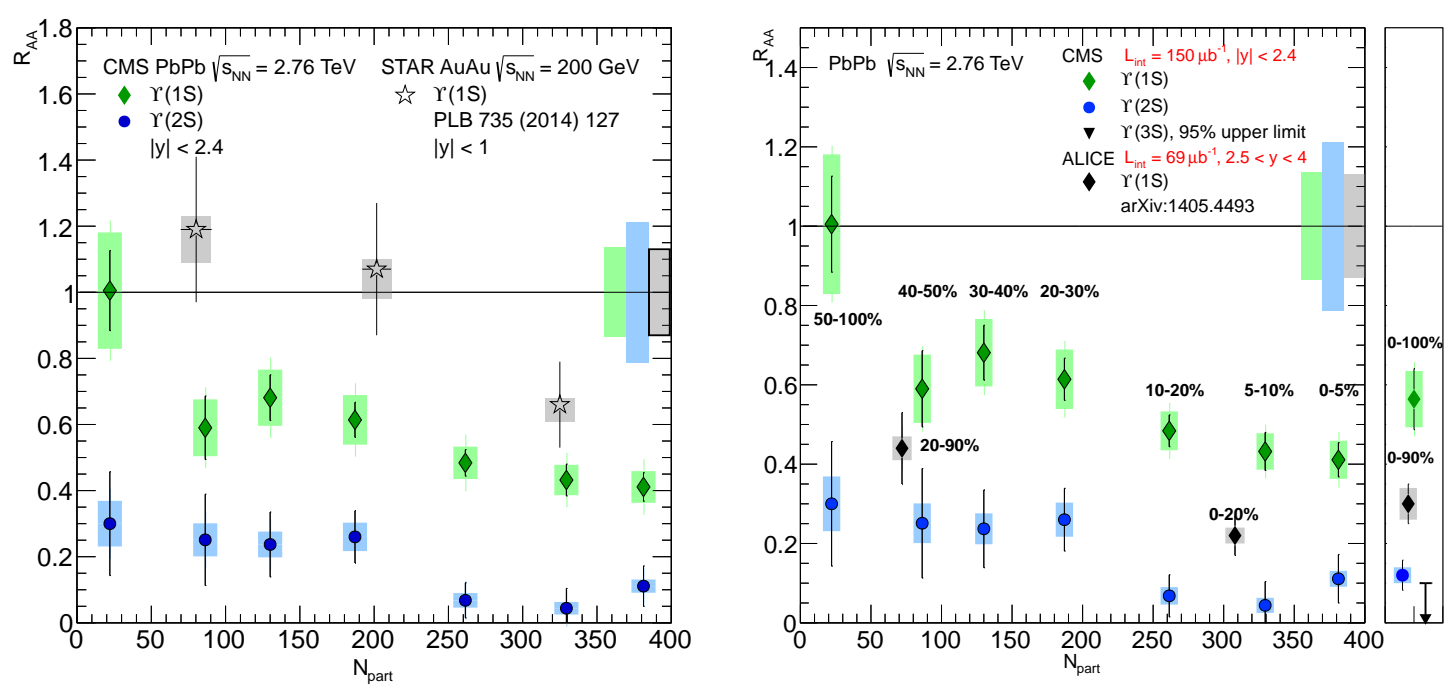

Figure 3: (left) Comparison of the $\Upsilon(1 \mathrm{~S})$ nuclear modification factor $R_{A A}$ centrality dependence result to the $\Upsilon(1 \mathrm{~S}) R_{A A}$ measurent by STAR [20]. The STAR Results are binned in classes of centrality 0-10\%, 10$30 \%$ and $30-60 \%$. (right) Comparison of the $\Upsilon(1 \mathrm{~S})$ and $\Upsilon(2 \mathrm{~S})$ nuclear modification factor $R_{A A}$ centrality dependence results to the $\Upsilon(1 \mathrm{~S}) R_{A A}$ measured in the forward rapidity range $(2.5<\mathrm{y}<4)$ by ALICE[21]

To understand different mechanism of suppression in hot and cold nuclear matter CMS utilizes proton-lead $(\mathrm{pPb})$ collision data provided by LHC in the start of 2013. This data provides an essential reference to understand initial state effects and may also provide insight into cold nuclear effects that may be distinct from the suppression effects observed in $\mathrm{PbPb}$ collisions $[18,19]$. Figure 4 (left) shows the ratios of the excited states, $\Upsilon(2 S)$ and $\Upsilon(3 S)$, to the ground state, $\Upsilon(1 S)$ in $\mathrm{pPb}$ collisions at $\sqrt{s_{N N}}=5.02 \mathrm{TeV}$ with respect to pp collisions at $\sqrt{s}=2.76 \mathrm{TeV}$. These ratios are compared to the corresponding ratios for $\mathrm{PbPb}$ (cross) collisions at $\sqrt{s_{N N}}=2.76 \mathrm{TeV}[19,22]$. Double ratios in $\mathrm{pPb}$ collision are larger than $\mathrm{PbPb}$ ratios but still are less than one. This suggest presence of final state effects in $\mathrm{pPb}$ and $\mathrm{PbPb}$ collisions, which affect more strongly excited states $\Upsilon(2 \mathrm{~S})$ and $\Upsilon(3 \mathrm{~S})$ than $\Upsilon(1 \mathrm{~S})$. The $\mathrm{pp}$ and $\mathrm{pPb}$ data are further analyzed separately as a function of event activity variables. Figure 4 (right) shows the excited to ground states cross section ratios, $\Upsilon(2 \mathrm{~S}) /(1 \mathrm{~S})$, as a function of number of charged particles in the rapidity range $|\eta|<2.4$ for all three collision systems. These ratios, are found to decrease with increasing charged-particle multiplicity. This trend can be explained in two opposite ways. If, on the one hand, the $\Upsilon(1 \mathrm{~S})$ is systematically produced with more particles than the excited states, it would influence the underlying distribution of charged particles and create an artificial effect when selected in small multiplicity bins. This effect should be sensitive to the underlying multiplicity distribution, and would result in a larger correlation if one reduces the size of the multiplicity bins. On the other hand, if the $\Upsilon$ are interacting with the surrounding environment, the $\Upsilon(1 \mathrm{~S})$ is expected, as the most tightly bound state and the one of smallest size, to be less affected than $\Upsilon(2 S)$ and $\Upsilon(3 S)$, leading to a decrease of the $\Upsilon(n S) / \Upsilon(1 S)$ ratios with increasing multiplicity. In either case, the ratios will continuously decrease from the pp 
to $\mathrm{pPb}$ to $\mathrm{PbPb}$ systems, as a function of event multiplicity.
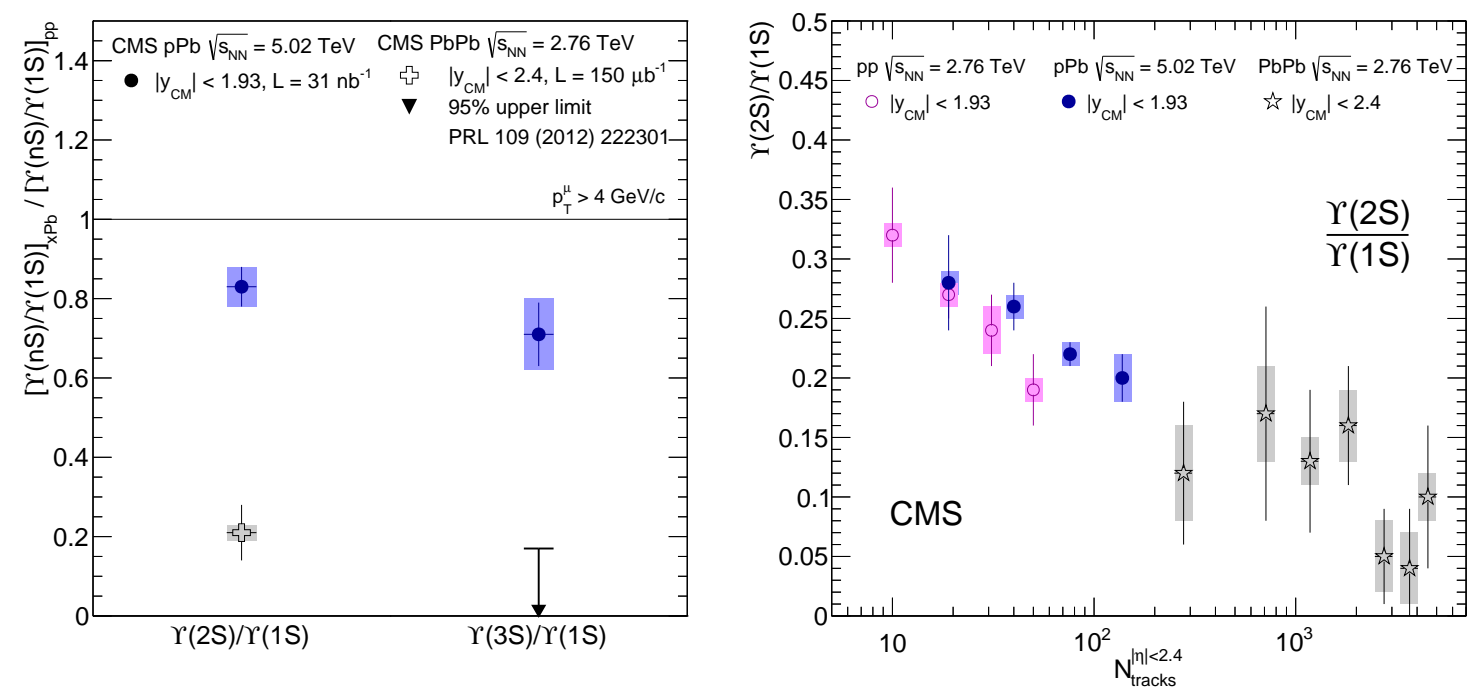

Figure 4: (left) Event activity integrated double ratios of the excited states, $\Upsilon(2 S)$ and $\Upsilon(3 S)$, to the ground state, $\Upsilon(1 \mathrm{~S})$ in $\mathrm{pPb}$ collisions at $\sqrt{s_{N N}}=5.02 \mathrm{TeV}$ with respect to pp collisions at $\sqrt{s}=2.76 \mathrm{TeV}$ (circles), compared to the corresponding ratios for $\mathrm{PbPb}$ (cross) collisions at $\sqrt{s_{N N}}=2.76 \mathrm{TeV}[19,22]$. (right) Single cross section ratios $\Upsilon(2 \mathrm{~S}) / \Upsilon(1 \mathrm{~S})$ for $\left|y_{\mathrm{CM}}\right|<1.93$ versus number of charged tracks measured in $|\eta|<2.4$ for pp collisions at $\mathrm{TeV} \sqrt{s}$ (open symbols), pPb collisions at $\sqrt{s_{N N}} 5.02 \mathrm{TeV}$ (closed symbols) and $\mathrm{PbPb}$ collisions at $\sqrt{s_{N N}} 2.76 \mathrm{TeV}$ (open stars).

\section{Heavy flavour measurements}

CMS offers B meson measurements via detecting secondary $\mathrm{J} / \psi$ coming from a displaced vertex. Figure 5 shows the $R_{A A}$ of $\mathrm{B}$ mesons via secondary $\mathrm{J} / \psi$ compared to $R_{A A}$ of light hadrons $[12,13]$. We can conclude that at high $p_{T}>10 \mathrm{GeV} / c$ the suppression of $\mathrm{B}$ mesons and light hadrons are consistent, but at low $p_{T} \mathrm{~B}$ meson $R_{A A}$ is larger as compared to light hadrons. Combining CMS B meson results with the ALICE measurements of D-meson [23] containing c-quarks it follows that at low $p_{T}$ there is mass hierarchy in the amount of suppression such that, $R_{\mathrm{AA}}^{\text {light hadrons }}<$ $R_{A A}^{\mathrm{D} \text { meson }}<R_{A A}^{\mathrm{B} \text { meson }}$. Several theoretical models claims to get similar mass scaling if they include both collisional as well as radiative energy loss [24, 25].

\section{Summary}

With the recent LHC measurements combined with RHIC measurements an overall understanding of quarkonia and heavy flavour production in heavy ion collisions is emerging. One of the the most noticeable results is sequential suppression of $\Upsilon$ states observed first time in heavy ion collisions. The $\Upsilon$ suppression at LHC is more than that at RHIC showing that the matter at LHC has stronger colour screening. The measurements of $\Upsilon$ states in $\mathrm{pPb}$ collisions suggest the presence of final effects in $\mathrm{pPb}$ collisions affecting ground state and excited states differently. 


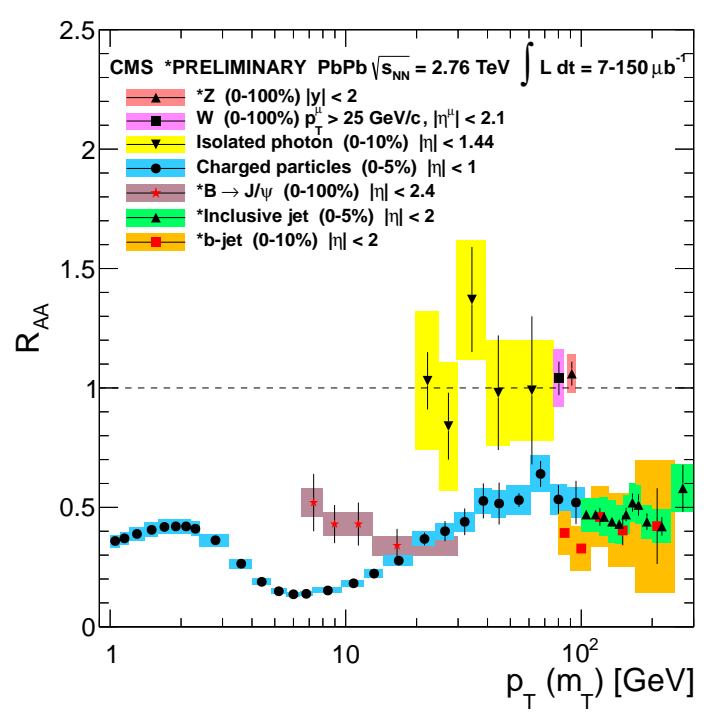

Figure 5: Nuclear modification factor $(R)$ of B mesons via secondary $\mathrm{J} / \psi$ compared to $R_{A A}$ of light charged hadrons $[12,13]$.

High $p_{T} \mathrm{~J} / \psi$ is more suppressed at LHC as compared to RHIC. The enhancement of low $p_{T}$ $\mathrm{J} / \psi$ as compared to RHIC hints that that there is substantial regeneration. The enhancement of ratio of yields of excited to ground state charmonia at low $p_{T}$ also points in this direction. More statistics expected in $\mathrm{PbPb}$ collisions at $5 \mathrm{TeV}$, a better $p_{T}$ and rapidity dependence of quarkonia will certainly quantify the effects of colour screening and regeneration.

The LHC hints mass hierarchy in suppression of hadrons below $p_{T} \sim 8 \mathrm{GeV} / \mathrm{c}$. For $p_{T}>10$ $\mathrm{GeV} / c$, the suppression of light hadrons, charm mesons and bottom mesons are consistent. Better precision and larger $p_{T}$ reach will help quantifying the energy loss properties of the medium.

\section{References}

[1] T. Matsui and H. Satz, “J/ $\psi$ Suppression by Quark-Gluon Plasma Formation,” Phys. Lett. B 178, 416 (1986).

[2] X. -M. Xu, D. Kharzeev, H. Satz and X. -N. Wang, "J/ $\psi$ suppression in an equilibrating parton plasma," Phys. Rev. C 53, 3051 (1996).

[3] V. Kumar, P. Shukla and R. Vogt, "Quarkonia suppression in PbPb collisions at $\sqrt{s_{N N}}=2.76 \mathrm{TeV}$," Phys. Rev. C 92, no. 2, 024908 (2015).

[4] A. Adare et al. [PHENIX Collaboration], " $J / \psi$ suppression at forward rapidity in Au+Au collisions at $\sqrt{s_{N N}}=200 \mathrm{GeV}$," Phys. Rev. C 84, 054912 (2011).

[5] N. Brambilla, S. Eidelman, B. K. Heltsley, R. Vogt, G. T. Bodwin, E. Eichten, A. D. Frawley and A. B. Meyer et al., "Heavy quarkonium: progress, puzzles, and opportunities," Eur. Phys. J. C 71, 1534 (2011).

[6] A. Andronic, P. Braun-Munzinger, K. Redlich and J. Stachel, "Statistical hadronization of charm in heavy ion collisions at SPS, RHIC and LHC,” Phys. Lett. B 571, 36 (2003). 
[7] B. Muller, J. Schukraft and B. Wyslouch, "First Results from Pb+Pb collisions at the LHC," Ann. Rev. Nucl. Part. Sci. 62, 361 (2012).

[8] P. Shukla [CMS Collaboration], "Overview of quarkonia and heavy flavour measurements by CMS," arXiv:1405.3810 [nucl-ex].

[9] R. Vogt, "Cold Nuclear Matter Effects on J/ $\psi$ and $\Upsilon$ Production at the LHC," Phys. Rev. C 81, 044903 (2010).

[10] R. Vogt, "Shadowing Effects on J/ $\psi$ and $\Upsilon$ Production at the LHC," arXiv:1507.04418 [hep-ph].

[11] S. Chatrchyan et al. [CMS Collaboration], “The CMS experiment at the CERN LHC," JINST 3, S08004 (2008).

[12] S. Chatrchyan et al. [CMS Collaboration], "Suppression of non-prompt $J / \psi$, prompt $J / \psi$, and Y(1S) in $\mathrm{Pb}+\mathrm{Pb}$ collisions at $\sqrt{s_{N N}}=2.76 \mathrm{TeV}$," JHEP 1205, 063 (2012).

[13] C. Mironov [CMS Collaboration], "Overview of results on heavy flavour and quarkonia from the CMS Collaboration,” Nucl. Phys. A 904-905, 194c (2013).

[14] Z. Tang [STAR Collaboration], “ $J / \psi$ production and correlation in $\mathrm{p}+\mathrm{p}$ and Au+Au collisions at STAR,” J. Phys. G 38, 124107 (2011).

[15] B. B. Abelev et al. [ALICE Collaboration], "Centrality, rapidity and transverse momentum dependence of $\mathrm{J} / \psi$ suppression in Pb-Pb collisions at $\sqrt{s_{N N}}=2.76 \mathrm{TeV}$," Phys. Lett. 743, 314 (2014).

[16] V. Khachatryan et al. [CMS Collaboration], "Measurement of Prompt $\psi(2 S) \rightarrow J / \psi$ Yield Ratios in $\mathrm{Pb}-\mathrm{Pb}$ and $p-p$ Collisions at $\sqrt{s_{N N}}=2.76$ TeV," Phys. Rev. Lett. 113, no. 26, 262301 (2014).

[17] CMS Collaboration, CMS Physics Analysis Summary CMS-HIN-12-001 (2012)

[18] S. Chatrchyan et al. [CMS Collaboration], "Indications of suppression of excited $\Upsilon$ states in $\mathrm{PbPb}$ collisions at $\sqrt{S_{N N}}=2.76$ TeV," Phys. Rev. Lett. 107, 052302 (2011).

[19] S. Chatrchyan et al. [CMS Collaboration], "Observation of sequential Upsilon suppression in $\mathrm{PbPb}$ collisions,” Phys. Rev. Lett. 109, 222301 (2012).

[20] L. Adamczyk et al. [STAR Collaboration], "Suppression of $\Upsilon$ production in d+Au and Au+Au collisions at $\sqrt{s_{N N}}=200 \mathrm{GeV}$," Phys. Lett. B 735, 127 (2014) [Phys. Lett. B 743, 537 (2015)].

[21] B. B. Abelev et al. [ALICE Collaboration], "Suppression of $\Upsilon(1 \mathrm{~S})$ at forward rapidity in $\mathrm{Pb}-\mathrm{Pb}$ collisions at $\sqrt{s_{\mathrm{NN}}}=2.76 \mathrm{TeV}$,” Phys. Lett. B 738, 361 (2014).

[22] S. Chatrchyan et al. [CMS Collaboration], "Event activity dependence of $\Upsilon(\mathrm{nS})$ production in $\sqrt{s_{N N}}=5.02 \mathrm{TeV} \mathrm{pPb}$ and $\sqrt{s}=2.76 \mathrm{TeV}$ pp collisions," JHEP 1404, 103 (2014).

[23] B. Abelev et al. [ALICE Collaboration], "Suppression of high transverse momentum D mesons in central Pb-Pb collisions at $\sqrt{s_{N N}}=2.76 \mathrm{TeV}$," JHEP 1209, 112 (2012).

[24] U. Jamil and D. K. Srivastava, "Nuclear suppression of heavy quark production at forward rapidities in relativistic heavy ion collisions," J. Phys. G 37, 085106 (2010).

[25] K. Saraswat, P. Shukla and V. Singh, "Constraining heavy quark energy loss using $B$ and $D$ meson measurements in heavy ion collision at RHIC and LHC energies, arXiv:1507.06742 [nucl-th]. 